ARTICLE

\title{
Nucleation and spreading of a heterochromatic domain in fission yeast
}

Michaela J. Obersriebnig ${ }^{1, \star}$, Emil M.H. Pallesen ${ }^{1, \star}{ }^{,}$Kim Sneppen ${ }^{2}$, Ala Trusina ${ }^{2} \&$ Geneviève Thon ${ }^{1}$

Outstanding questions in the chromatin field bear on how large heterochromatin domains are formed in space and time. Positive feedback, where histone-modifying enzymes are attracted to chromosomal regions displaying the modification they catalyse, is believed to drive the formation of these domains; however, few quantitative studies are available to assess this hypothesis. Here we quantified the de novo establishment of a naturally occurring $\sim 20-\mathrm{kb}$ heterochromatin domain in fission yeast through single-cell analyses, measuring the kinetics of heterochromatin nucleation in a region targeted by RNAi and its subsequent expansion. We found that nucleation of heterochromatin is stochastic and can take from one to ten cell generations. Further silencing of the full region takes another one to ten generations. Quantitative modelling of the observed kinetics emphasizes the importance of local feedback, where a nucleosome-bound enzyme modifies adjacent nucleosomes, combined with a feedback where recruited enzymes can act at a distance.

\footnotetext{
${ }^{1}$ Department of Biology, University of Copenhagen, BioCenter, Ole Maaløes Vej 5, 2200 Copenhagen, Denmark. ${ }^{2}$ Niels Bohr Institute, University of Copenhagen, Blegdamsvej 17, 2100 Copenhagen, Denmark. * These authors contributed equally to this work. Correspondence and requests for materials should be addressed to G.T. (email gen@bio.ku.dk).
} 
$\mathrm{M}$ any histone-modifying enzymes bind their modified substrates. This binding, together with other types of positive feedback that generally accompany it, is believed to facilitate the spreading of nucleosome modifications along chromosomes from nucleation sites and the modification of newly deposited nucleosomes following DNA replication, perhaps participating in epigenetic memory. In these models, an enzyme attracted to a modified nucleosome modifies nucleosomes in the vicinity. In contrast to the molecular interactions that lead to the deposition of histone modifications, kinetic aspects of chromatin formation that would allow testing such models have seldom been reported.

A well-documented example of positive feedback is in heterochromatin formation, where methyltransferases of the KMT1 family bind methylated histone H3K9 (H3K9me) with chromodomains and methylate H3K9 with their SET domains ${ }^{1-3}$, establishing a major type of repressive heterochromatin whose dynamics are critical to development, cell differentiation and the maintenance of genome integrity ${ }^{4-13}$. In the fission yeast Schizosaccharomyces pombe, mutations in the chromodomain of the KMT1 Clr4 that reduce the affinity of $\mathrm{Clr} 4$ for $\mathrm{H} 3 \mathrm{~K} 9 \mathrm{me}$ alleviate $\mathrm{H} 3 \mathrm{~K} 9 \mathrm{me}$ in vivo, underscoring the importance of $\mathrm{Clr} 4$ self-recruitment for heterochromatin formation ${ }^{2,3,14,15}$. There, self-recruitment is fostered by several histone deacetylases (HDACs), RNA interference (RNAi) factors and an $\mathrm{H} 3 \mathrm{~K} 4$ demethylase (Lid2) that together indirectly promote $\mathrm{H} 3 \mathrm{~K} 9$ methylation by Clr4 (refs 3,15-22). Likewise, antagonistic histone-modifying enzymes benefit from positive feedback. Histone acetyltransferases, which antagonize HDACs, bind to acetylated nucleosomes via bromodomains, to promote expressed acetylated chromatin states ${ }^{23}$. Demethylases that remove methyl groups from $\mathrm{H} 3 \mathrm{~K} 9$ me bind to nucleosomes bearing active state modifications via double Tudor or PHD domains, or participate in histone-binding protein complexes. This is the case for JMJD2A ${ }^{24,25}$, PHF8 (ref. 26), ceKDM7A ${ }^{27}$ and PHF2 (ref. 28) in higher eukaryotes, and for S. pombe Lsd1 (refs 29-31).

Chromatin landscapes are dynamically shaped by such opposing activities at all times, with widespread changes in histone modification profiles underlying changes in gene expression programmes ${ }^{4-13}$. Not surprisingly, temporal transition states as occur during cell differentiation, early embryogenesis or at the onset of disease have been more difficult to characterize experimentally than terminally differentiated chromatin, because some changes affect only small numbers of cells or do not occur synchronously in populations. Here, to gain insights into the kinetics of chromatin formation, we applied single-cell measurements to capture the non-equilibrium states of a naturally occurring $\mathrm{H} 3 \mathrm{~K} 9 \mathrm{me}$ domain of $S$. pombe as the domain transitions from euchromatin to heterochromatin.

In S. pombe, all detectable H3K9me depends on Clr4 (ref. 2,32). $\mathrm{Clr} 4$ is found in the $\mathrm{ClrC}$ complex, which catalyses the formation of large heterochromatic domains at centromeres, telomeres and in the mating-type region (Zhang et al. ${ }^{3}$ and references therein). The system has been extensively studied. Among its many advantages is the fact that fission yeast lacks DNA methylation on cytosines, eliminating a common caveat in investigations of the role of histone modifications in epigenetic inheritance.

In the region used for our measurements, the mating-type region, heterochromatin occupies $\sim 20 \mathrm{~kb}$ between the two boundary elements $I R-L$ and $I R-R^{33,34}$ (Fig. 1), silencing the mat2- $P$ and mat3- $M$ mating-type loci and the entire intervening region $^{34-37}$. RNAi is believed to nucleate heterochromatin formation through an $\sim 4$-kb element located between mat2-P and mat3-M, cenH. RNAi contributes strongly to heterochromatin formation at $S$. pombe centromeres ${ }^{38,39}$ where many repeats homologous to $c e n H$ are found ${ }^{40}$ and, to a lesser extent, at telomeres where a few repeats are found ${ }^{41}$. Non-coding RNA transcribed from the repeats are amplified by an RNA-dependent RNA polymerase, cleaved by Dicer into small interfering RNAs, and incorporated into an Argonaute family protein, Agol, in the RITS complex. Multiple physical interactions take place between RNAi complexes and $\mathrm{ClrC}$, leading to the recruitment of $\mathrm{ClrC}$ to regions where RNAi is active and, conversely, to the recruitment of RNAi to regions with H3K9me, in self-inforcing loops $3,20-22,42,43$.

Deletion of RNAi factors do not result in immediately discernable heterochromatin loss in the mating-type region, unlike for centromeres ${ }^{38,44,45}$, but the de novo heterochromatin formation in the mating-type region is slow in RNAi mutants ${ }^{44-47}$ and $c e n H$ mediates heterochromatin formation at ectopic locations in an RNAi-dependent manner ${ }^{44,48}$, indicating RNAi acting at cenH catalyses the establishment of heterochromatin. In addition to RNAi, the Atf1/Pcrl dimer binding to a site between $c e n H$ and mat3- $M$ also contributes to heterochromatin formation ${ }^{49}$. Mutants lacking $c e n H$, the $\Delta K$ mutants, adopt a bistable phenotype alternating between two epigenetic states, one state with the same defects as $c l r 4 \Delta$ mutants and the other state like wild type $\mathrm{e}^{50,51}$. Switches between the two states occur at rates of $\sim 1 / 2,000$ cell divisions ${ }^{51}$. Together with the phenotypes of RNAi mutants and with the many ties established between RNAi and $c e n H$ repeats elsewhere in the genome, the bistability of $\Delta K$ strains suggests that deletion of cenH reduces the rate of heterochromatin establishment by removing an RNAi-dependent element that normally facilitates Clr4 recruitment. RNAi would increase the rate of establishment from $\sim 1 / 2,000$ cell division in cells lacking $c e n H$ to some higher rate that we set up to measure.

The in vivo kinetic measurements presented here were conducted with the goal of quantitative modelling. The notion that modified nucleosomes promote inheritance and spreading of modifications by attracting modifying enzymes is common in the literature but tests of these models have been hampered by a lack of quantitative data. Our measurements allow us to test conditions for which positive feedback in the recruitment of histone-modifying enzymes permits heterochromatin formation and inheritance, expanding on our previous modelling ${ }^{52-55}$ to uncover basic principles underlying eukaryotic chromatin formation. Specifically, our new data and modelling show how combined local reactions, where an enzyme recruited to a nucleosome modifies the neighbour nucleosomes, and global reactions, where some recruited enzymes modify more distant nucleosomes, contribute to the establishment of large heterochromatic domains. The reactions result in long-lasting heterogeneity in cell populations that are in the process of changing epigenetic state.

\section{Results}

Heterochromatin is established slowly in populations. Reporter genes inserted between the $I R-L$ and $I R-R$ boundaries are silenced by heterochromatin as shown in Fig. 1 for an insertion of $\mathrm{ura}^{+}$ near mat2-P, (XbaI)::ura4 ${ }^{+}$(ref. 37). (XbaI)::ura $4^{+}$cells do not grow in the absence of uracil and are resistant to 5-fluoroorotic acid, a toxigenic substrate of the orotidine $5^{\prime}$-phosphate decarboxylase encoded by $\mathrm{ura4}^{+}$. This silencing is alleviated in mutant cells lacking Clr4 (ref. 37). We monitored the establishment of Clr4-mediated silencing by re-introducing a functional $\mathrm{clr}^{+}$gene into (XbaI)::ura4 ${ }^{+}$clr44 cells through a genetic cross. (XbaI):: ura ${ }^{+}$clr4 4 cells were crossed with mat1-P 17::LEU2 clr4 ${ }^{+}$cells whose mating-type region is easy to follow in crosses. Spores were micromanipulated into a grid on rich medium, allowed to form colonies and colonies were 
a

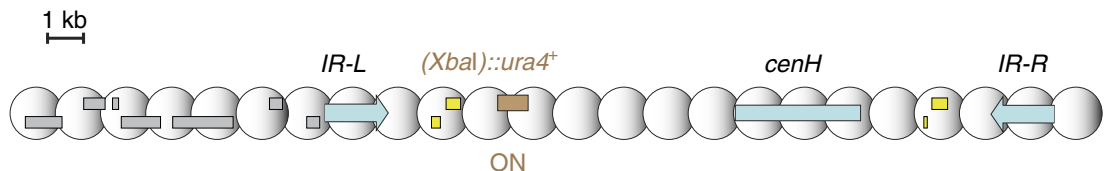

clr4s

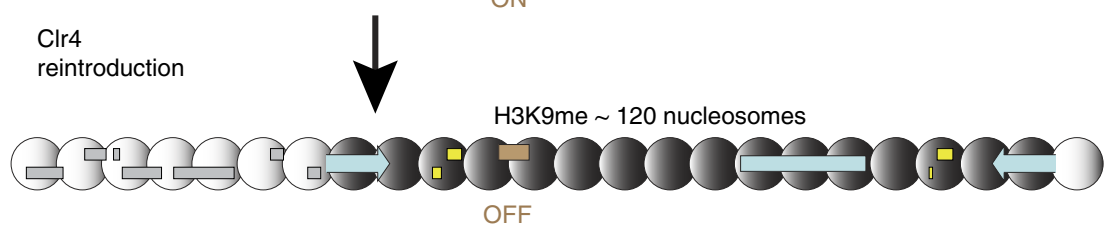

b
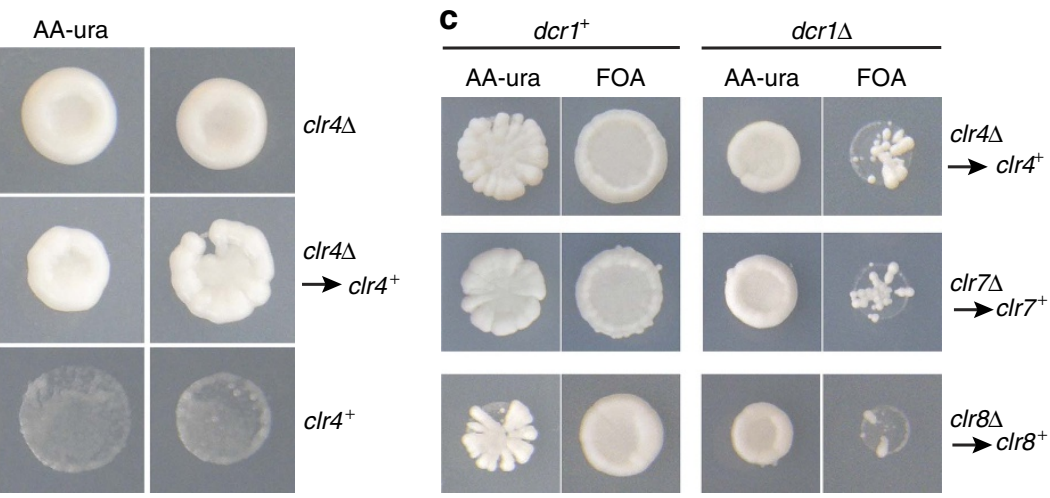

Figure 1 | Slow establishment of heterochromatic silencing by the H3K9 methyltransferase Clr4. (a) Mating-type region. Nucleosomes between the IR- $L$ and $I R-R$ boundaries are methylated by $\mathrm{Clr} 4$, silencing gene expression. The chromosomal location of the $(X b a l):: u r a 4^{+}$reporter is shown. cenH: $\sim 4.2 \mathrm{~kb}$ region with centromere homology; grey boxes: ORFs; yellow boxes: silent mating-type information at mat2- $P$ and mat3-M. (b) de novo silencing of (Xbal)::ura $4^{+}$by $\mathrm{Clr} 4$. Colonies formed by spores on rich medium were replicated onto medium lacking uracil. All colonies shown contain the (Xbal):: ura $^{+}{ }^{+}$reporter. A phenotypic gap is observed following re-introduction of $c l r 4^{+}$into $\mathrm{clr} 44$ cells (middle panels) allowing clr $4^{+}$cells to grow in the absence of uracil. The three colonies shown on the last row $\left(\mathrm{clr} 4^{+}\right)$are genetically identical to the colonies above them (clr44 to $\left.\mathrm{clr} 4^{+}\right)$but their heterochromatin is fully established. (c) Dicer facilitates the establishment of heterochromatic silencing by Clr4, Clr7 and Clr8. Wild-type alleles were re-introduced into deletion strains by genetic crosses as indicated. Silencing of (Xbal)::ura ${ }^{+}$permits growth on 5-fluoroorotic acid (FOA). Establishement of (Xbal):: ura4 ${ }^{+}$silencing is more efficient in the $d c r 1^{+}$than in the $d c r 14$ progeny of each cross.

replicated onto selective media to assign genotypes and to assay (XbaI):: $\mathrm{rra}^{+}{ }^{+}$expression. As expected, (XbaI)::ura4${ }^{+}$remained derepressed in the (XbaI):: $u r a 4^{+}$clr4 4 progeny of the cross (Fig. 1b; first row). Surprisingly, (XbaI)::ura ${ }^{+}$also remained derepressed in the (XbaI)::ura4 ${ }^{+} \mathrm{clr}^{+}$progeny of the cross (Fig. 1b; second row). In a control cross where a (XbaI)::ura4 ${ }^{+}$ $\mathrm{clr}^{+}$strain with fully established heterochromatin was used instead of the (XbaI):::ura4 ${ }^{+}$clr44 strain, (XbaI)::ura4 ${ }^{+}$ remained repressed in all progeny, indicating meiosis does not alleviate repression (Fig. lb; third row). In the $\mathrm{clr}^{+}$re-introduction experiment, some cells still displayed a mutant Ura ${ }^{+}$ phenotype $>20$ generations after they became genetically wild type (Fig. 1b; second row). This long phenotypic gap indicates that $\mathrm{H} 3 \mathrm{~K} 9 \mathrm{me}$ is not established efficiently, certainly not at each cell division, in a naive mating-type region lacking all H3K9me.

We compared establishment of silencing of (XbaI)::ura4 ${ }^{+}$ following re-introduction of $c l r 4^{+}$in $d c r 1^{+}$and $d c r 1 \Delta$ cells. A similar experiment was conducted previously in $d c r 1 \Delta$ cells, where slow establishment was observed; however, the experiment did not examine a wild-type $d c r 1^{+}$background for comparison, rendering it difficult to judge the extent to which lack of RNAi slows down establishment ${ }^{44}$. Here, genetic crosses were conducted in two ways that produced identical results. First, a $\mathrm{clr}^{+}$re-introduction experiment was performed in a $d c r 1 \Delta$ background, where both parents were $d c r 1 \Delta$, and compared with a parallel cross in which both parents were $d c r 1^{+}$. Second, (XbaI)::ura4 ${ }^{+}$clr4 4 cells were crossed with $c l r 4^{+} d c r 1 \Delta$ cells and the (XbaI):: $\mathrm{ura4}^{+}{ }^{\mathrm{clr}}{ }^{+} d c r 1 \Delta$ progeny, lacking RNAi, were compared with the (XbaI)::ura4 ${ }^{+}$clr4 ${ }^{+} d c r 1^{+}$progeny, proficient for RNAi. Delayed establishment of 5-fluoroorotic acid resistance and uracil auxotrophy in the dcrld progeny (Fig. 1c) showed that heterochromatic silencing following re-introduction of $\mathrm{Clr} 4$ is slowed down by the absence of RNAi. Further, two subunits of the ClrC complex, respectively, Clr7 (a.k.a. Raf2 or Dos2) and Clr8 (a.k.a. Raf1 or Dos1), behaved as Clr4 in re-introduction experiments in both $d c r 1^{+}$and $d c r 1 \Delta$ backgrounds (Fig. 1c). In addition, the wild-type levels of matingtype switching and sporulation were also acquired with a delay in $\mathrm{clr}^{+}$re-introduction experiments, consistent with slow heterochromatin establishment in both RNAi-proficient and -deficient cells, with a more pronounced delay in RNAi mutants (Supplementary Fig. 1). These results confirm that RNAi normally facilitates the establishment of $\mathrm{H} 3 \mathrm{~K} 9 \mathrm{me}$ in the mating-type region by the $\mathrm{ClrC}$ complex as already shown by others $^{45,46}$. Importantly, these results also show that the RNAimediated establishment of heterochromatin in wild-type cells is very inefficient. Slow establishment in a wild-type background has, to our knowledge, not been reported previously, because previous studies examined establishment at later time points following the re-introduction of $c l r 4^{+}$.

Stochastic establishment and clonal inheritance. To assess the behaviour of individuals in populations of cells undergoing heterochromatin establishment, we inserted a gene encoding the yellow fluorescent protein (YFP) near mat3-M ((EcoRV)::YFP; Figs 2 and 3a). As a reference, we inserted a gene for the fluorescent protein mCherry in euchromatin, at the ura4 locus 
a
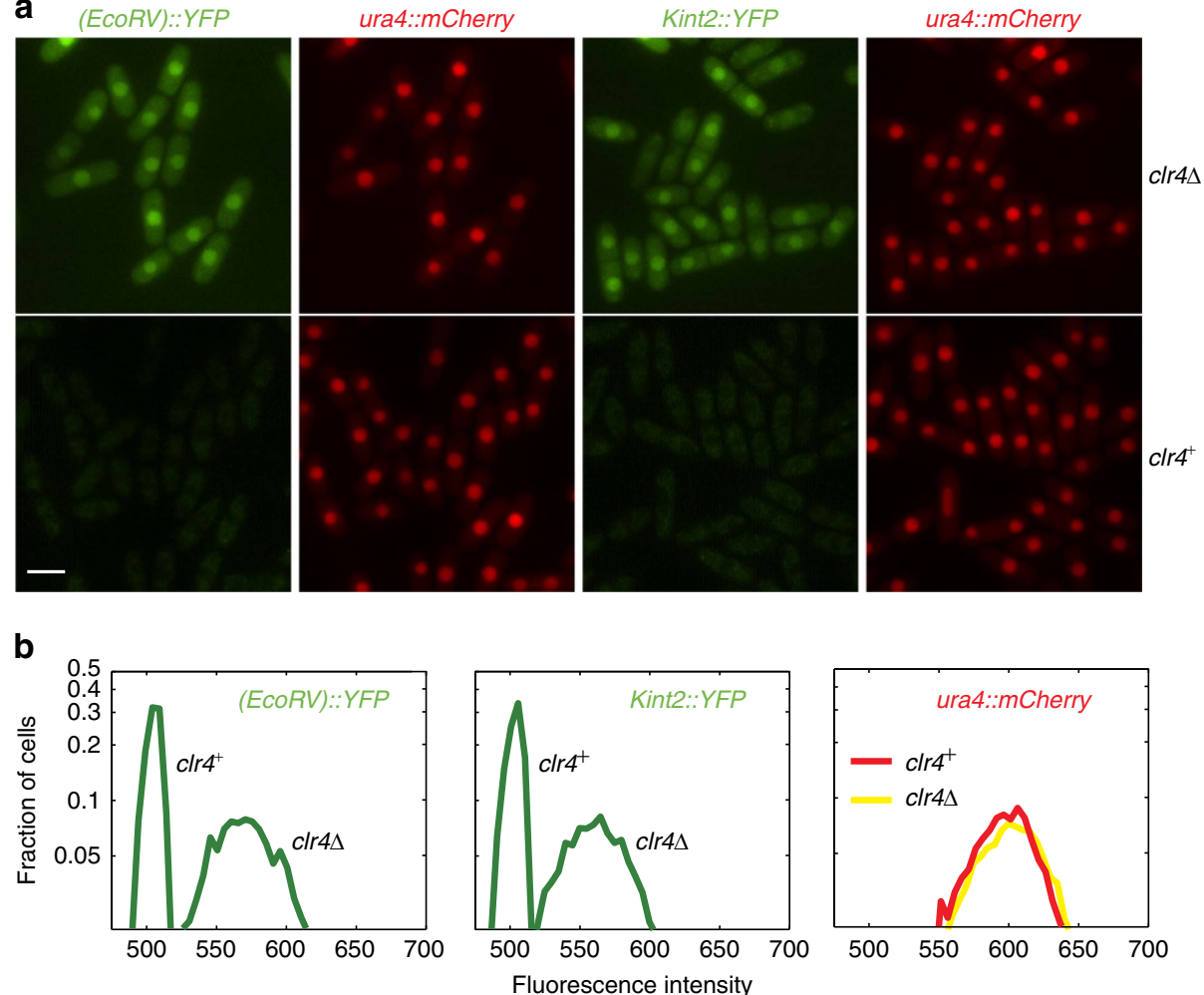

Figure 2 | Development of fluorescent reporters for single-cell measurements. A nuclear-targeted YFP under control of the ura4 ${ }^{+}$promoter was expressed from two sites in the mating-type region, respectively, (EcoRV) near the centromere-distal edge of the heterochromatic region and kint 2 within the cenH element. The location of these sites is depicted in Figs 3 and 4. mCherry was expressed in the same cells from the ura4 ${ }^{+}$euchromatic locus. (a) Fluorescence of kint2::YFP, (EcoRV)::YFP and ura4::mCherry in clr4 4 and clr $4^{+}$cells. Scale bar, $5 \mu \mathrm{m}$. (b) Distribution of fluorescence intensities for the same strains, obtained by measuring nuclear fluorescence in microscopy images.

(ura4::mCherry). Expression of both YFP and mCherry was controlled by the ura4 promoter and terminator of transcription, and both proteins were targeted to the nucleus by an SV40 nuclear localization sequence. This experimental setup is similar to the setup used in Saccharomyces cerevisiae by $\mathrm{Xu}$ et al. ${ }^{56}$ As was the case for the (XbaI)::ura4 ${ }^{+}$reporter used in Fig. 1, $(E c o R V):: Y F P$ was repressed by heterochromatin in wild-type cells but it was expressed in a clr4 $\Delta$ mutant (Fig. 2). In clr $4 \Delta$ cells, a unimodal fluorescence distribution was observed for YFP, the distribution did not overlap with the fluorescence distribution in clr4 ${ }^{+}$cells. The expression of ura4::mCherry did not differ between $\mathrm{clr}^{+}$and $\mathrm{clr} 4 \Delta$ cells (Fig. 2).

As for $(\mathrm{XbaI}):: \mathrm{ura}^{+}$, the expression of $(E c o R V):: Y F P$ was assayed following re-introduction of the wild-type $c l r 4^{+}$gene through a genetic cross but, unlike for $(\mathrm{XbaI}):: \mathrm{ura}^{+}{ }^{+}$, cells could be interrogated starting at their first division following the re-introduction of $\mathrm{clr} 4^{+}$. Prototrophic markers were used to select the desired progeny. $\arg 12^{+}$was used to select $\mathrm{clr} 4^{+}$and $\mathrm{ura}^{+}$integrated at the $\mathrm{XmnI}$ site on the euchromatic side of $I R-L^{51}$ was used to select the mating-type region with $(E c o R V):: Y F P . \quad(E c o R V):: Y F P \quad c l r 4^{+}$spores were induced to germinate by placing the spores at $33^{\circ} \mathrm{C}$ in minimal medium. Germination occurs in a synchronous manner under these conditions. It produces cells that can be examined right away in a temperature-controlled growth chamber under a fluorescence microscope (Fig. 3), or allowed to proliferate in liquid cultures with frequent sampling (Fig. 4). Establishment of H3K9me in the mating-type region resulted in silencing of YFP. Time-lapse series were obtained in which germinated spores divided to form microcolonies, examples of which are shown in Fig. $3 \mathrm{~b}$ and Supplementary Movies 1-3. These series indicated that silencing of YFP occurred stochastically at a rate of $\sim 1$ in 8 cell divisions (for example, 9 events in 72 divisions of 'ON' cells in Supplementary Movies). Once established, the silenced state was transmitted to the mitotic progeny. Crowding imposed a limit to the number of generations for which cells could be imaged in the growth chamber. Liquid cultures were used instead, to examine cells for longer periods following spore germination (Fig. 4). Growth curves were established and the proportion of cells expressing YFP was determined every few hours by fluorescence microscopy, confirming the estimated rate of $(E c o R V):: Y F P$ silencing. Although some YFP protein is likely to be transmitted from one generation to the next, the protein is diluted exponentially as cells keep dividing. Time-lapse microscopy, where it is possible to trace back turn-off points to single cells in pedigrees, indicates that this transmission has little effect on the estimated rate of silencing. Using a homologous reporter system in $S$. cerevisiae, $\mathrm{Xu}$ et al. ${ }^{56}$ showed that similar measurements were independent of reporter stability.

The silencing kinetics of $(E c o R V):: Y F P$ indicate that the establishment of heterochromatin results from rare stochastic events rather than from slow changes building up gradually over many generations in a synchronous manner for all cells in the population. A slow and steady increase would lead to a large frequency of concomitant switches, which were not observed. Although low, the rate of establishment at $(E c o R V)$ in wild type is $\sim 150$-fold higher than in the $\Delta K$ mutant $^{51}$.

Establishment within the RNAi-dependent cenH element. We investigated whether the establishment of heterochromatin at the proposed target for $\mathrm{RNAi}$, cen $\mathrm{H}$, differs from neighbouring region 
a

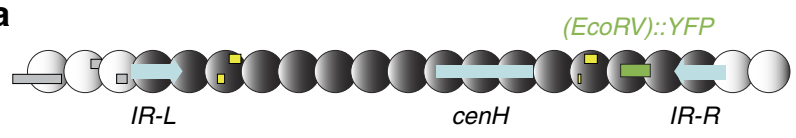

IR-L

cenH

IR-R
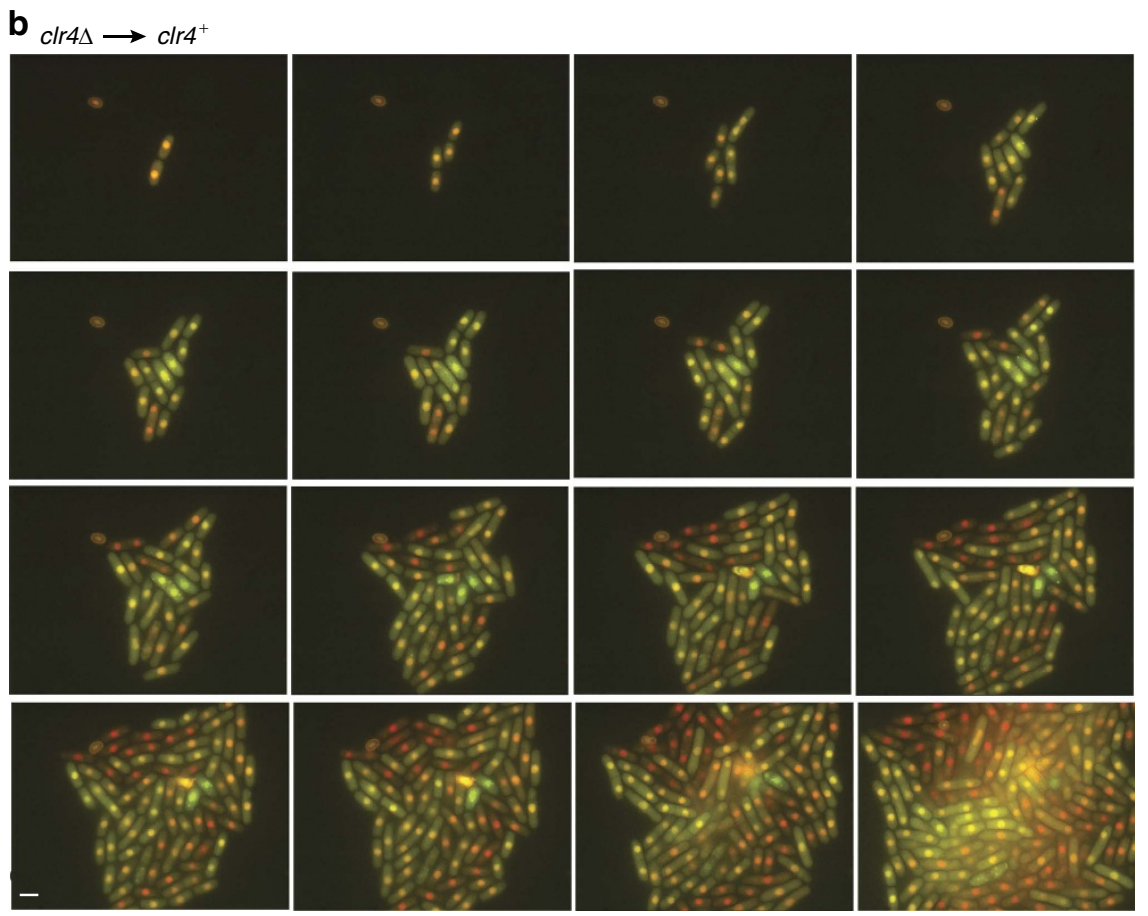

Figure 3 | Fission yeast cells dividing under a fluorescence microscope establish gene silencing. (a) Fluorescent reporters used for imaging. (b) Overlay of the mCherry and YFP channels, starting at the first cell division following the re-introduction of clr $4^{+}$through a cross. At first, cells express both YFP and mCherry (yellow-green signal). As they divide, a few cells silence YFP (red signal). The silenced state is inherited clonally in their progeny. Scale bar, $5 \mu$ m. Additional timepoints and separate channels are shown in Supplementary Movies 1-3.

with a strain where YFP was expressed from cenH (Kint2::YFP) instead of $(E c o R V):: Y F P$ (Figs 2 and 4). In clr4 4 cells, YFP expression was similar at Kint2 and (EcoRV) (Fig. 2). Following re-introduction of Clr4, silencing at Kint2::YFP occurred faster than silencing at $(E c o R V):: Y F P$ measured in a parallel experiment (Fig. 4), indicating that heterochromatin might be first established within $c e n H$, and that the modifications would subsequently spread to the rest of the region.

Heterochromatin spreads outwards from cenH. Whether Kint2 and $(E c o R V)$ are silenced consecutively was investigated with strains with two linked fluorescent reporters. One strain contained the mCherry gene at the Kint 2 site within cenH and the YFP gene at (EcoRV) near mat3-M (Fig. 5a). The other strain had the reversed order of markers. Both YFP and mCherry were controlled by the $\mathrm{ura}^{+}$promoter and terminator of transcription, and the proteins were targeted to the nucleus. In clr4 $\Delta$ cells, YFP and mCherry were expressed from both locations and the YFP (respectively mCherry) signal was of similar intensity whether expressed from Kint 2 or from (EcoRV) (Fig. 5a). clr4 ${ }^{+}$was re-introduced as above through a cross where only the $c l r 4^{+}$ progeny with the labelled mating-type region were allowed to germinate and the expression of YFP and mCherry was monitored over time by fluorescence microscopy, sampling liquid cultures (Fig. 5b-h). Silencing occurred with the same kinetics as observed previously (Fig. 4 and Supplementary Movies 1-3). The rates of silencing were determined by fitting an exponential function (Matlab) to the number of 'ON' cells as a function of time. The \pm values correspond to the $95 \%$ confidence interval resulting from fitting. The rates of silencing at Kint2 were $0.20 \pm 0.04$ silencing event per cell division for YFP and $0.25 \pm 0.02$ for mCherry. The rates of silencing at $(E c o R V)$ were $0.12 \pm 0.02$ measured with mCherry or $0.14 \pm 0.02$ for YFP. Silencing at $(E c o R V)$ occurred with a delay compared with Kint2 and, with rare exceptions, only in cells that had also silenced the reporter at Kint2 (Fig. 5b). Five generations after re-introduction of $\mathrm{clr}^{+}$, for example, $\sim 50 \%$ of cells expressed both reporters, $\sim 15 \%$ had silenced both reporters, $\sim 35 \%$ had silenced Kint 2 but not $(E c o R V)$ and $<1 \%$ had silenced $(E c o R V)$ but not Kint2. The same kinetics were observed for the Kint2::YFP (EcoRV)::mCherry strain (where YFP was silenced before mCherry) and for the Kint2::mCherry (EcoRV)::YFP strain (where mCherry was silenced before YFP), ruling out that different fluorescent properties or protein half-lives might cause the differences observed for the two locations (Fig. 5). At each time point the probability of silencing $(E c o R V)$ was much higher (in the order of 100-fold higher) in cells that had silenced Kint2 than in cells that had not silenced Kint2, indicating that the two events are not independent, and that heterochromatin spreads from Kint2 to (EcoRV). Together, these observations show that the establishment of heterochromatic silencing occurs first at $c e n H$, and that nucleation of heterochromatin at cenH facilitates heterochromatin formation in the rest of the mating-type region.

Quantitative modelling of heterochromatin formation. We previously modelled how modified nucleosomes can participate in epigenetic memory by attracting read-write enzymes that catalyse the modifications they display ${ }^{52-54}$. Here we used core 

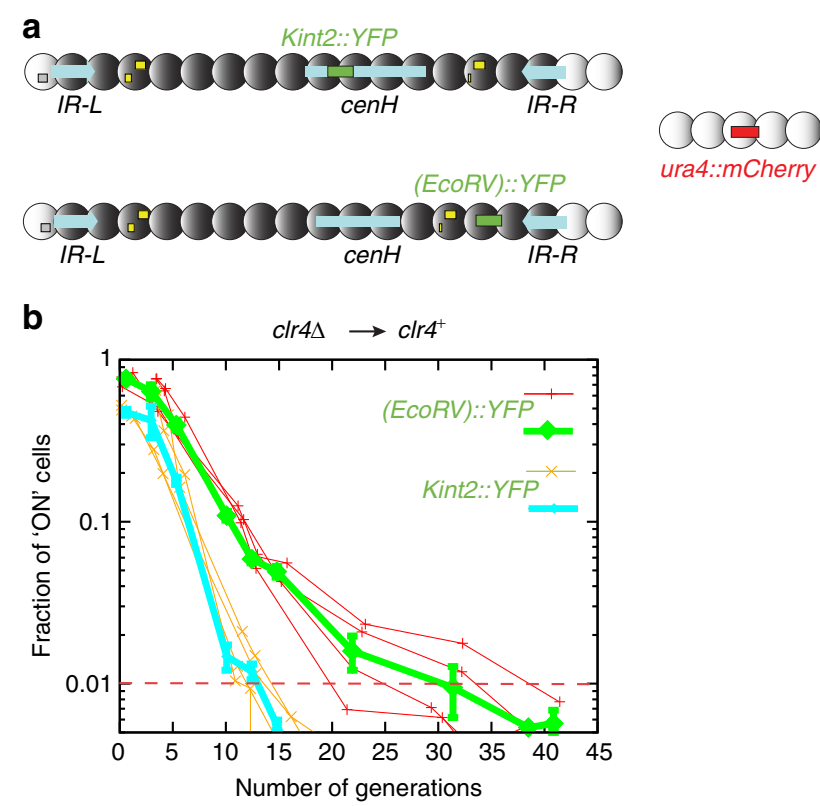

Figure 4 | The kinetics of heterochromatin establishment are faster within cenH than outside cenH. (a) Location of the kint2::YFP and (EcoRV)::YFP reporters used in the experiment. These reporters are also shown in Fig. 2. (b) Establishment of heterochromatic silencing at kint2::YFP and (EcoRV)::YFP in re-introduction experiments, starting at the first division following re-introduction of $\mathrm{Clr}^{+}{ }^{+}$. Fluorescence intensity was measured in microcopy images sampling liquid cultures at the indicated time point. The experiment was conducted in quadruplicate (thin lines) and the data were binned (thick lines). Error bars represent the s.d. of the values in each bin.

elements from Dodd et al. ${ }^{52}$ to model heterochromatin establishment in wild-type cells, introducing the notion that RNAi facilitates Clr4 recruitment within cenH (Fig. 6). In this modified model, the mating-type region is represented by 140 nucleosomes arranged in a line. As in Dodd et al. ${ }^{52}$ nucleosomes exist in three states: unmodified (U), heterochromatic (methylated at H3K9; M) or euchromatic (acetylated; A). Nucleosomes are exposed to modifying enzymes that may be recruited or be spontaneously acting as shown in Fig. 6a. Changes in chromatin structure result from (1) iterating the 'nucleosome modification procedure' described below and (2) periodically mimicking cell division by replacing half the nucleosomes in the region with unmodified nucleosomes.

In the nucleosome modification procedure ${ }^{52}$, a randomly picked 'modifying' nucleosome is allowed to interact with a 'substrate' nucleosome. No change is made if the two nucleosomes are in the same state, otherwise the substrate nucleosome is moved one step towards the state of the modifying nucleosome with a rate reflecting the ability of nucleosomes to directly or indirectly recruit histone-modifying enzymes. In addition, nucleosomes may be exposed to modifying enzymes that act independently of the other nucleosomes within the considered system. All reactions are assigned rate parameters.

The system is iterated in discrete events using a Gillespie algorithm with the assigned rate parameters. Each selected event defines one attempted modification process. In case the event is a spontaneous conversion, for example, of $\mathrm{A}$ to $\mathrm{U}$, one selects one nucleosome randomly and makes the conversion if the nucleosome is in the appropriate state (in this case an A state). In case the chosen reaction is a read-write event (for example, M-statecatalysed conversion of $\mathrm{A}$ to $\mathrm{U}$ ), one randomly selects a potential modifying nucleosome and a target nucleosome. If this pair of nucleosomes is not in states that permit the chosen recruitment (for example, if the recruiting nucleosome is not in $\mathrm{M}$ ), no change is made to the system. If both recruiting and target nucleosomes match the chosen reaction, the target nucleosome is modified.

Two modes of recruitment interactions are permitted, local or global $^{53}$. For local feedback, an enzyme recruited by a modified nucleosome can modify only the immediate neighbours of that nucleosome. Hence, only nucleosomes that are adjacent to the modifying nucleosome can be picked to function as substrates. For global feedback, the enzyme recruited by a modified nucleosome can modify any nucleosome in the system with a probability inversely proportional to the distance between the two nucleosomes. In this case, any nucleosome in the system can be picked as a potential substrate, according to the chosen distance probability rule.

Noticeably, the attempt rate is not the same as the success rate, because a reaction is only successful when the chosen pair of nucleosomes are in states that match the assigned process. For example, when the number of $\mathrm{A}$ or $\mathrm{M}$ is small, the actual number of reactions where $M$ facilitates a change from $A$ to $U$ is small. Therefore, this particular reaction will mostly occur when the system is in the transition between an A-dominated state and an M-dominated state. In the Supplementary Notes 1-3 we describe model variants and simulations in detail.

In the version of the model shown in Fig. 6 only M-statecatalysed conversion of A to $U$ was considered global, whereas the three other processes were local. Each process was attempted at a rate of 100 times per nucleosome between cell divisions, except the $\mathrm{M}$-state-catalysed $\mathrm{U}$ to $\mathrm{M}$ conversion that was attempted 30 times per nucleosome. In addition to these recruited reactions, we allowed small rates of spontaneous changes (2.5 per nucleosome per generation for all conversions) that represent chance encounters with histone-modifying enzymes external to the system.

To reflect the ability of cenH to facilitate $\mathrm{H} 3 \mathrm{~K} 9 \mathrm{me}$, a source of modification was introduced at cenH, consisting of 29 nucleosomes for which spontaneous conversions of $A$ to $U$ were attempted at a rate of 16 attempts per nucleosome per cell generation. This source together with the recruitment rules described above recapitulated the kinetics of heterochromatin establishment within and outside cenH (Fig. 6b-d). Following removal of the source, the model correctly reproduced the bistability of the remaining system of 70 nucleosomes (Fig. 6e), that is, the model also recapitulates the phenotypes of the $\Delta K$ mutants.

The above model is one example of a wider class of recruitment scenarios for nucleosome-mediated epigenetics. Supplementary Note 3 explores variant models where not all modification reactions are catalysed by read-write enzymes, models with other combinations of local and global recruitments, and also models with more than three nucleosome states. Apart from the need for long-range recruitment reactions and cooperativity formulated by Dodd et $a .^{52}$, the combination of model testing and our new timing experiments teaches us the following: (i) Recruitments on all modifications greatly enhance the robustness of epigenetic bistability against spontaneous conversions. (ii) Most recruitment reactions should be acting only locally, to reproduce the sequential silencing of the two reporters. (iii) Within three-state models, one needs long-range recruitment on the $A$ to $U$ modification acting from the silenced $M$ state, to obtain the observed combination of robust bistability (to spontaneous conversions) and differential timing (between cenH and the whole system). (iv) The four-state model provides a doubleswitch interpretation for the two consecutive silencing events.

The model variation with additional modification steps deserves special attention. It is justified by the fact that each 
a

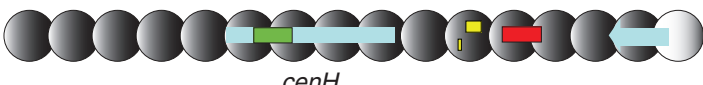

cenH
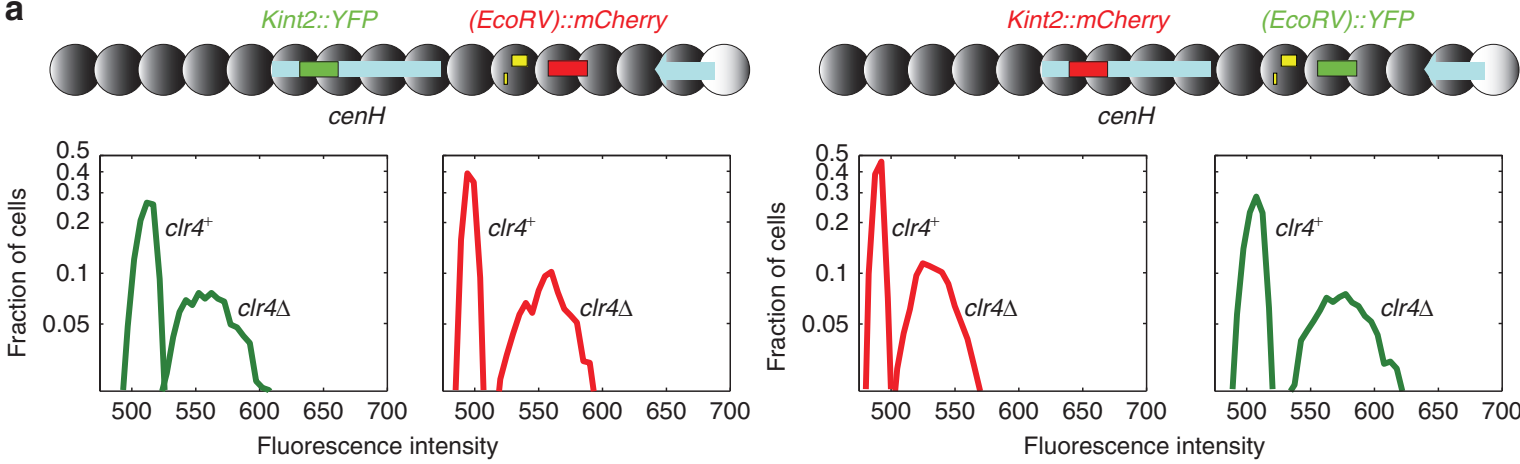

b
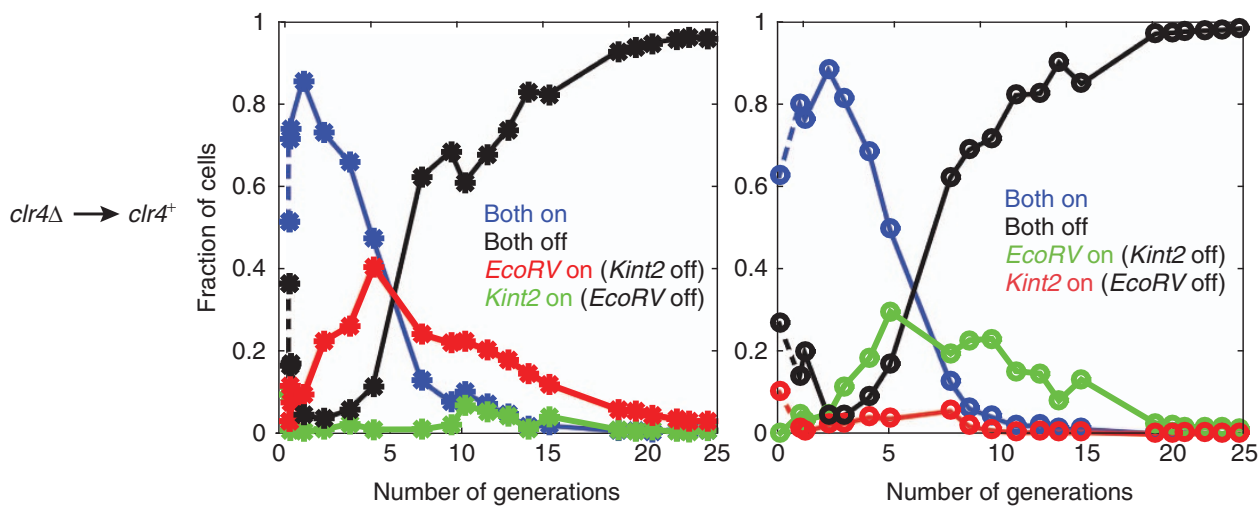
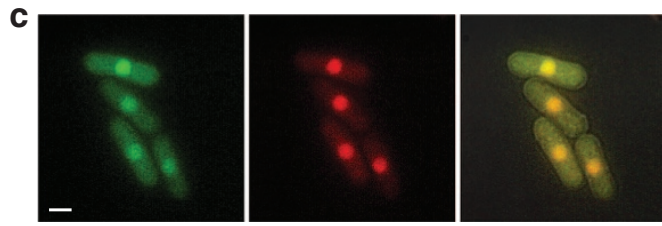

e
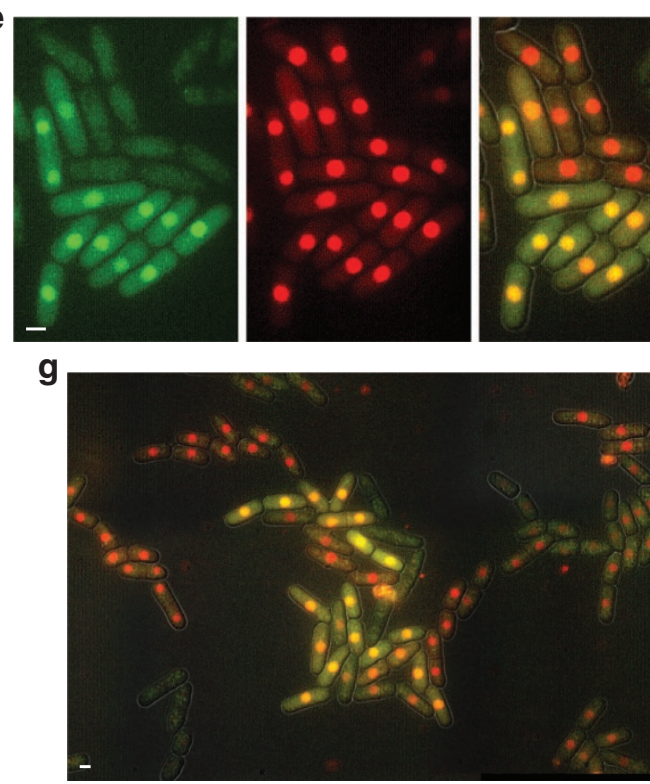
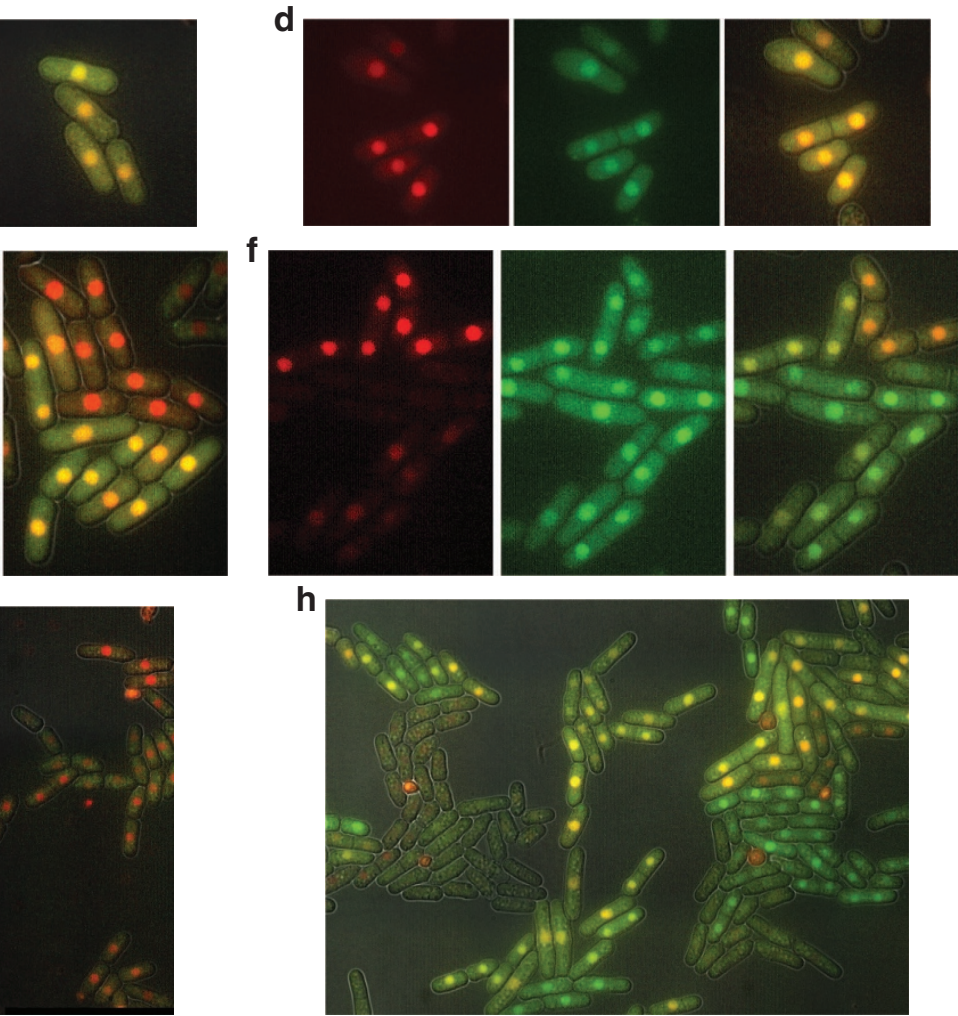

Figure 5 | Spreading of heterochromatin outward from cenH. (a) Fluorescent reporters used in the experiment. Cells with Kint2::YFP (EcoRV)::mCherry are shown on the left in all panels; Kint2::mCherry (EcoRV)::YFP cells are shown on the right. (b) YFP and mCherry fluorescence was measured by microscopy at the indicated time points following the re-introduction of $\mathrm{Clr}^{+}{ }^{+}$examining $>1,000$ cells for each data point. Some uncertainty for the initial timepoint, due to the difficulty in counting germinating spores, is indicated by dashed lines. Histograms for the fluorescence distributions are shown in Supplementary Fig. 2. (c-h) Representative images. (c,d) At the second division following spore germination, the vast majority of clr $4^{+}$cells express both reporters. (e,f) As cells divide (generation 5), silencing of the reporter at Kint2 (whether Kint2::YFP on the left or Kint2::mCherry on the right) precedes silencing of the reporter at (EcoRV). (d,f) Generation 7. Scale bars, $2 \mu \mathrm{m}$. 

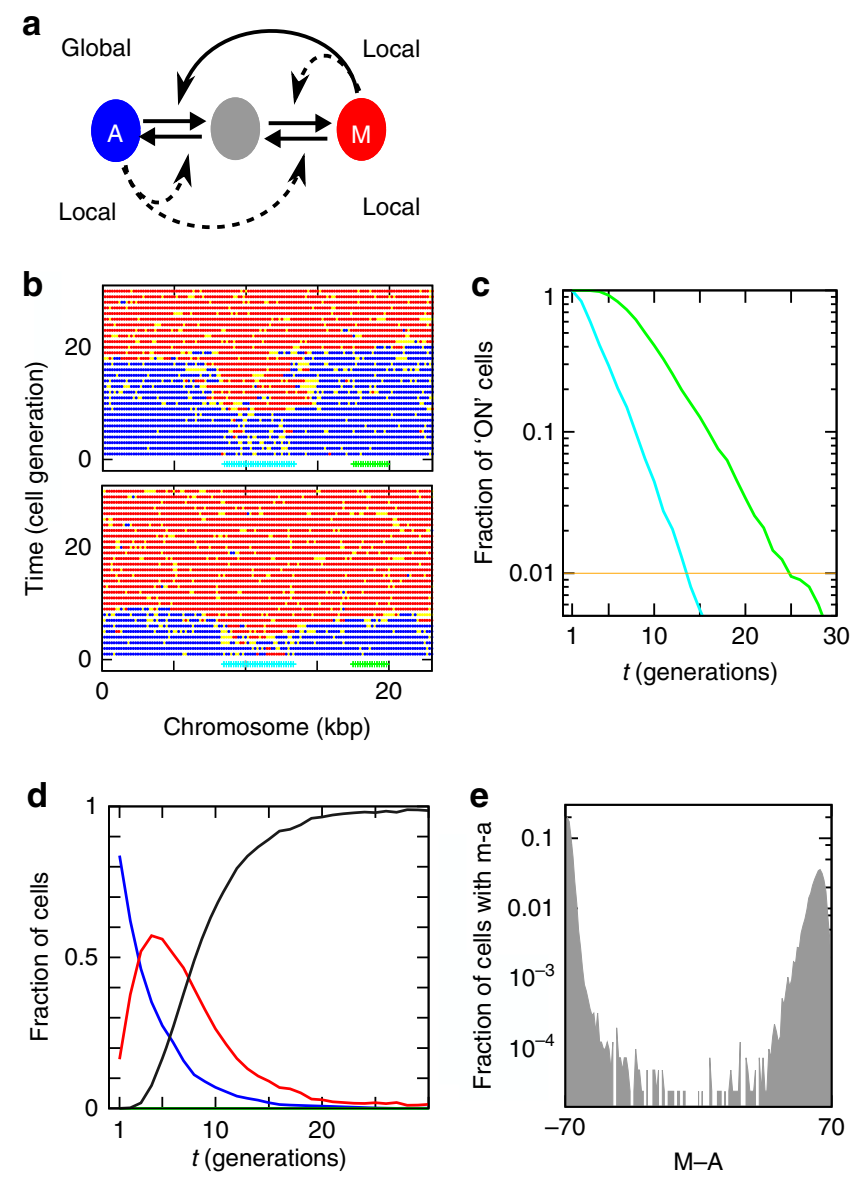

Figure 6 | Combinations of global and local nucleosomal cross-talks recapitulate the observed kinetics. (a) Positive feedbacks in the recruitment of histone-modifying enzymes. Nucleosomes exist in three states, methylated ( $M$; red), unmodified ( $U$; grey), or acetylated ( $A$; blue). Methylated and acetylated nucleosomes recruit modifying enzymes capable of modifying the immediate neighbours of the nucleosome to which they bind (thin dashed arrows: local feedback) and, for $A$ to $U$, more distant nucleosomes as well (thick arrow: global feedback). The evolution of a $23-k b$ region representing the mating-type region with two reporters was simulated, using parameters and a source of methylation at cenH (cyan) as described in the text. (b) Two model simulations with identical parameters of the dynamics in nucleosome modification profile over the 23-kb matingtype region following the re-introduction of $\mathrm{Clr} 4$ at time 0 . (c) Times of silencing for the Kint2 (cyan) and (EcoRV) (green) positions in the simulations occurred with the kinetics measured in vivo at these two locations. (d) Fraction of cells with none, one or both genes active as function of time. (e) Distribution of epigenetic states for the $\Delta K$ mutant with an epigenetic stability compatible with experimental measurements.

nucleosome contains two copies of each histone, that each lysine can be multiply methylated and also that the binding of proteins to methylated nucleosomes may be cooperative, as proposed in the case of Swi6 (ref. 57). Further, the various methylation states of $\mathrm{H} 3 \mathrm{~K} 9$ in vivo have different affinities for chromodomain proteins 3,58 . We find that a fourth step provides increased robustness to non-recruited modifications (see Supplementary Note 4). A second and remarkable outcome of the four-state model is an alternative interpretation of the spreading of silencing marks where spreading occurs intermittently from cen $H$ to the remaining system. Silencing of cenH represents one stochastic switch, followed by a second switch that provides substantial cellto-cell heterogeneity in the time needed to silence the whole region. The experimental hallmark of such a second switch is an exponential distribution of the time difference between the first and the second silencing event.

\section{Discussion}

Although the molecular interaction networks that permit the association of histone-modifying enzymes to chromatin are often well known, kinetic parameters are not. Here we determined the kinetics of de novo establishment of heterochromatin in the mating-type region of fission yeast. We found that establishment occurs stochastically in populations. Stochastic establishment and epigenetic inheritance result in heterogeneous populations where distinct epigenetic states coexist for $\sim 20$ generations. Mathematical modelling, used to test conditions for which positive feedback in the recruitment of histone-modifying enzymes to chromatin recapitulates the observed rates of heterochromatin nucleation and spreading, provides new insights into the mechanisms of heterochromatin formation.

A first point that modelling helps understand is the large cellto-cell variation in the time of heterochromatin establishment. Conceivably, the establishment of a chromatin state in a large chromosomal domain results from initiating events followed by spreading that lead to a stable conversion of the entire region. The observed stochasticity of establishment might reflect a switch-like silencing of cenH followed by a stochastic propagation. Our model shows how propagation relying on the recruitment of histone-modifying enzymes by modified nucleosomes would result in stochastic establishment with a characteristic timescale of a few cell generations. Heterogeneity in gene expression and chromatin structure is observed in populations of differentiating cells (reviewed by, for example, Tee and Reinberg ${ }^{59}$ ). Our observations indicate how heterogeneity resulting from cells engaging into diverse differentiation programmes might be compounded by temporal stochasticity in the establishment of chromatin structures. The stochastic establishment of H3K9me chromatin domains might in itself be a key determinant of some differentiation programmes, as it has been proposed to underlie important cell-fate decisions in mammals (reviewed in Magklara and Lomvardas ${ }^{13}$ ). Stochasticity in the establishment of chromatin structures might generally contribute to developmental variation and diversity in adult populations and it might account for the incomplete penetrance of some genetic defects where only a fraction of the population is affected by a mutation resulting in an otherwise severe phenotype (for example, Morgan et $\left.a l .{ }^{60}\right)$. Cell-to-cell heterogeneity in the nucleation of H3K27me3 at the Arabidopsis FLC locus ensures a quantitative response to cold exposure at the population level ${ }^{61,62}$, which, as for the present system, is epigenetically stored in cis in the chromosomes of individual cells ${ }^{50,51,63}$.

Mechanistically, variations on our model highlight the complementary effects of global and local nucleosomal recruitments in chromatin formation. Locally acting recruitments reflect steric constraints for reactions in which a nucleosome-bound enzyme might not be able to catalyse the modification of a distant nucleosome, while globally acting recruitments account for reactions that might not be similarly constrained. Here we found that combinations of global and local effects were necessary to model the observed combination of nucleation rate and subsequent kinetics of spreading. Feedback interactions limited to local contacts, as proposed for the modelling of a smaller artificial system ${ }^{64}$ could not account for the observed exponential distribution of turn-off times at the cen $H$ region. The $\sim 5-15 \mathrm{~h}$ it subsequently takes to spread along the 25 nucleosomes between cenH to EcoRV is about a factor ten faster than the 0.18 nucleosomes per hour observed by Hathaway et al. ${ }^{64}$ in a 
mammalian system with its much longer cell generation. Thus, in units of cell generation the timescale for the spreading part of the dynamics might be comparable between these different organisms. However, the large cell-to-cell variation in the time of spreading is compatible with an alternative scenario of intermittent dynamics that has a characteristic timescale of about five generations. The possibility for this scenario is in particular supported by the four-state variant of our model (Supplementary Note 4).

It has been pointed out before that purely global feedback interactions would not permit the confinement of chromatin states to specific chromosomal domain ${ }^{53}$. On the other hand, strictly local interactions fail to produce bistable systems $\mathrm{s}^{52,53}$ and they do not lead to the characteristic plateau shape of large heterochromatin domains ${ }^{64}$. In contrast, combinations of global and local recruitment allow the formation of bounded domains ${ }^{53}$ and account for the diverse kinetics of heterochromatin nucleation, spreading and inheritance we observed, including the behaviour of bistable mutants (Fig. 6).

Molecular mechanisms for both local and global feedback have been documented experimentally. In the case of $\mathrm{Clr} 4$ and for the mouse methyltransferases GLP and G9a, binding to trimethylated H3K9 stimulates the methylation of nearby nucleosomes in vitro ${ }^{65,66}$. Structural studies have also shown the importance of steric effects for proteins that are both readers and writers of histone modifications ${ }^{26,27}$. In the system we modelled, global positive feedback might be provided by RNAi spreading heterochromatin through transcription and associations with $\mathrm{RNA}^{49,67}$, through the polymerization of Tas3, a RITS subunit ${ }^{68}$ or by the association of ClrC with DNA polymerases ${ }^{69}$. As the association of HDACs with H3K9me domains can occur indirectly, through chromodomain proteins capable of multimerization ${ }^{18,19}$, we imagine that HDACs are likely to participate in global feedback reactions.

Long blocks of heterochromatin in plants and metazoans are structurally similar to the heterochromatic domains of $S$. pombe and it is known for some of them that their formation depends on KMT1 methyltransferases. Large H3K9me domains are formed in the olfactory receptor, zinc finger and protocadherin gene clusters during mammalian development, at multiple loci in cells that differentiate or adapt to growth conditions ${ }^{4-7,13}$ and on the inactive $\mathrm{X}$ chromosome in mammals (reviewed by Chaligné and $\mathrm{Heard}^{8}$ ). $\mathrm{H} 3 \mathrm{~K} 9 \mathrm{me}$ is also deposited at transposable elements in the Drosophila germline ${ }^{9}$ and, in the very early stages of mouse embryogenesis, as DNA methylation is erased and reprogrammed, the establishment of $\mathrm{H} 3 \mathrm{~K} 9$ methylation silences retroelements and modulate their ability to control gene activity through regional changes in chromatin structure ${ }^{10-12}$. Although widely different pathways might contribute to the formation of these heterochromatic domains, some basic mechanistic principles relying on the evolutionarily conserved ability of modified nucleosomes to recruit histone-modifying enzymes are likely to be shared. Indeed, modelling developed for S. pombe can be applied to other organisms ${ }^{61,62,70}$. Here we show how transient signals can help establish large heterochromatic domains by facilitating the self-recruitment of histone-modifying enzymes and how the signals can later become dispensable for heterochromatin maintenance. In the case at hand, a strong source was provided by RNAi, but other mechanisms could be substituted for RNAi.

Moreover, our observations document the existence of a Clr4-dependent epigenetic memory in fission yeast heterochromatin. Clearly, wild-type cells do not undergo heterochromatin establishment at each division, indicating the existence of an epigenetic component. The epigenetic mark would be lost in clr4 4 cells. The mark could be H3K9me itself or a more complex structure including proteins and RNA molecules associated with the heterochromatic region. Two recent studies have presented evidence that artificially established $\mathrm{H} 3 \mathrm{~K} 9$ me domains can be epigenetically maintained in $S$. pombe in the absence of the initiating sequence-specific recruitment ${ }^{14,15}$. In a different system, Polycomb complexes in the early Drosophila embryo, it was proposed that histone-modifying enzymes rather than the marks themselves are passed on during DNA replication ${ }^{71}$. In S. pombe, interactions between the Clr4 complex and replication factors ${ }^{69}$ might help Clr4 stay associated with chromatin behind the replication fork and/or interactions between $\mathrm{Clr} 4$ and protein complexes bound to non-coding RNAs might help maintain heterochromatin as the DNA replication fork passes through.

\section{Methods}

Strains. S. pombe strains were constructed using standard molecular biology and genetics as described in Supplementary Information. Genotypes are shown in Supplementary Table 1. Oligonucleotides are shown in Supplementary Table 2.

Re-introduction experiments. The S. pombe strain PG3437 was used to reintroduce $\mathrm{clr}^{+}{ }^{+}$into the clr44 strains SPK450, SPA26 and PG3404, and for a control cross with the $c l r 4^{+}$strain PG1789, SOM10 was used to re-introduce $c_{l r} 4^{+}$into SOM12 and SOM54, and PG3716 to re-introduce $c l r 4^{+}$into SOM67 and SOM70. Cells were propagated in yeast extract medium (YES), mixed pairwise on SPA plates supplemented with leucin, uracil and adenine, and incubated at $25^{\circ} \mathrm{C}$ for 2 days to induce mating and sporulation. The mating-mixes mixes were scraped, resuspended in 1-4 $\mathrm{ml} 1 \%$ glusulase (Roche) in $\mathrm{H}_{2} \mathrm{O}$, incubated overnight at $33^{\circ} \mathrm{C}$ and vortexed for $5 \mathrm{~min}$ to obtain preparations of random spores. Random spores were pelleted by centrifugation and washed in distilled $\mathrm{H}_{2} \mathrm{O}$. For each cross, 90 spores were arrayed on a rich medium plate (YES) by micromanipulation, incubated for 3 days at $33^{\circ} \mathrm{C}$ and spore colonies were replica-plated onto selective media to determine the genotypes of the progeny and, when applicable, to assay the expression of $\mathrm{ura4}^{+}$. Spores destined for fluorescence microscopy were resuspended in EMM2 and incubated for $5 \mathrm{~h}$ at $33^{\circ} \mathrm{C}$, at which point prototrophic spores started to germinate. The spores were placed in a home-made growth chamber in EMM2 medium for immediate microscopy or inoculated into $1 \mathrm{ml}$ EMM2 and incubated in a $13-\mathrm{ml}$ culture tube at $33^{\circ} \mathrm{C}$ with shaking. Spore germination was synchronous under these conditions. Liquid cultures were imaged at first every $\sim 2 \mathrm{~h}$ and less frequently for late timepoints. Cells were counted with a haemocytometer at each timepoint and an appropriate dilution was made in EMM2 to keep cultures in exponential phase. Cultures were plated on YES plates at the end of each experiment and replica-plated onto selective media to test the effectiveness of the selection.

Fluorescence microscopy. Cells were imaged with a Nikon Ti Eclipse epifluorescent microscope equipped with a $\times 100$ objective, an Andor Luca camera, a temperature-controlled enclosure and the NIS Elements acquisition programme. The fluorophores were visualized using a Yellow GFP BP HYQ filter set for YFP and a TRITC HYQ filter set for mCherry. Image acquisition times were $500 \mathrm{~ms}$ for YFP and $150 \mathrm{~ms}$ for mCherry.

Image Analyses. Fluorescent signals were quantified using a custom-image processing software written in MATLAB. In Figs 2 and 4, the Clr4-independent mCherry signal produced by ura4:mCherry was used to outline the boundaries of cell nuclei; average YFP intensities were measured within these boundaries. We found a clear separation in the YFP intensity (around 525, shown in Fig. 2) of silenced and non-silenced cells. This was then used to automatically count silenced and non-silenced cells. In Fig. 5, cell boundaries were identified using $z$-stacked bright-field images and custom-image processing software. The nucleus was found by either mCherry or YFP signal, or, in case where both reporters were silenced, the middle of the cell was taken to be the centre of the nucleus. The initial timepoints where the automatic cell identification was challenging due to the presence of spores, were quantified by manually defining a point in each nucleus, around which nuclear YFP and mCherry intensities were measured. The results of the automated image-processing software were tested against manually sampled intensities and against choices of silenced versus non-silenced cells made by visual inspection at the intermediate time-point, $\sim 5$ generations. For each sample and at each timepoint, the intensity of at least 1,000 cells was quantified.

\section{References}

1. Rea, S. et al. Regulation of chromatin structure by site-specific histone H3 methyltransferases. Nature 406, 593-599 (2000).

2. Nakayama, J., Rice, J. C., Strahl, B. D., Allis, C. D. \& Grewal, S. I. Role of histone H3 lysine 9 methylation in epigenetic control of heterochromatin assembly. Science 292, 110-113 (2001). 
3. Zhang, K., Mosch, K., Fischle, W. \& Grewal, S. I. Roles of the Clr4 methyltransferase complex in nucleation, spreading and maintenance of heterochromatin. Nat. Struct. Mol. Biol. 15, 381-388 (2008).

4. O'Geen, H. et al. Genome-wide analysis of KAP1 binding suggests autoregulation of KRAB-ZNFs. PLoS Genet. 3, e89 (2007).

5. Wen, B., Wu, H., Shinkai, Y., Irizarry, R. A. \& Feinberg, A. P. Large histone H3 lysine 9 dimethylated chromatin blocks distinguish differentiated from embryonic stem cells. Nat. Genet. 41, 246-250 (2009).

6. Hawkins, R. D. et al. Distinct epigenomic landscapes of pluripotent and lineage-committed human cells. Cell Stem Cell 6, 479-491 (2010).

7. Zhu, J. et al. Genome-wide chromatin state transitions associated with developmental and environmental cues. Cell 152, 642-654 (2013).

8. Chaligne, R. \& Heard, E. X-chromosome inactivation in development and cancer. FEBS Lett. 588, 2514-2522 (2014).

9. Sienski, G., Donertas, D. \& Brennecke, J. Transcriptional silencing of transposons by Piwi and maelstrom and its impact on chromatin state and gene expression. Cell 151, 964-980 (2012).

10. Gifford, W. D., Pfaff, S. L. \& Macfarlan, T. S. Transposable elements as genetic regulatory substrates in early development. Trends Cell Biol. 23, 218-226 (2013).

11. Matsui, T. et al. Proviral silencing in embryonic stem cells requires the histone methyltransferase ESET. Nature 464, 927-931 (2010).

12. Schlesinger, S. \& Goff, S. P. Retroviral transcriptional regulation and embryonic stem cells: war and peace. Mol. Cell. Biol. 35, 770-777 (2015).

13. Magklara, A. \& Lomvardas, S. Stochastic gene expression in mammals: lessons from olfaction. Trends Cell Biol. 23, 449-456 (2013).

14. Audergon, P. N. et al. Epigenetics. Restricted epigenetic inheritance of H3K9 methylation. Science 348, 132-135 (2015).

15. Ragunathan, K., Jih, G. \& Moazed, D. Epigenetics. Epigenetic inheritance uncoupled from sequence-specific recruitment. Science 348, 1258699 (2015).

16. Verdel, A. et al. RNAi-mediated targeting of heterochromatin by the RITS complex. Science 303, 672-676 (2004).

17. Li, F. et al. Lid2 is required for coordinating $\mathrm{H} 3 \mathrm{~K} 4$ and $\mathrm{H} 3 \mathrm{~K} 9$ methylation of heterochromatin and euchromatin. Cell 135, 272-283 (2008).

18. Motamedi, M. R. et al. HP1 proteins form distinct complexes and mediate heterochromatic gene silencing by nonoverlapping mechanisms. Mol. Cell 32, 778-790 (2008).

19. Fischer, T. et al. Diverse roles of HP1 proteins in heterochromatin assembly and functions in fission yeast. Proc. Natl Acad. Sci. USA 106, 8998-9003 (2009).

20. Gerace, E. L., Halic, M. \& Moazed, D. The methyltransferase activity of Clr4Suv39h triggers RNAi independently of histone H3K9 methylation. Mol. Cell 39, 360-372 (2010).

21. Bayne, E. H. et al. Stcl: a critical link between RNAi and chromatin modification required for heterochromatin integrity. Cell 140, 666-677 (2010).

22. Rougemaille, M. et al. Ers1 links HP1 to RNAi. Proc. Natl Acad. Sci. USA 109, 11258-11263 (2012).

23. Owen, D. J. et al. The structural basis for the recognition of acetylated histone $\mathrm{H} 4$ by the bromodomain of histone acetyltransferase gen5p. EMBO J. 19, 6141-6149 (2000).

24. Huang, Y., Fang, J., Bedford, M. T., Zhang, Y. \& Xu, R. M. Recognition of histone $\mathrm{H} 3$ lysine- 4 methylation by the double tudor domain of JMJD2A. Science 312, 748-751 (2006).

25. Wilson, J. R. Targeting the JMJD2A histone lysine demethylase. Nat. Struct. Mol. Biol. 14, 682-684 (2007).

26. Horton, J. R. et al. Enzymatic and structural insights for substrate specificity of a family of jumonji histone lysine demethylases. Nat. Struct. Mol. Biol. 17, 38-43 (2010).

27. Yang, Y. et al. Structural insights into a dual-specificity histone demethylase ceKDM7A from Caenorhabditis elegans. Cell Res. 20, 886-898 (2010).

28. Wen, H. et al. Recognition of histone H3K4 trimethylation by the plant homeodomain of PHF2 modulates histone demethylation. J. Biol. Chem. 285, 9322-9326 (2010).

29. Gordon, M. et al. Genome-wide dynamics of SAPHIRE, an essential complex for gene activation and chromatin boundaries. Mol. Cell. Biol. 27, 4058-4069 (2007).

30. Lan, F. et al. S. pombe LSD1 homologs regulate heterochromatin propagation and euchromatic gene transcription. Mol. Cell 26, 89-101 (2007).

31. Opel, M. et al. Genome-wide studies of histone demethylation catalysed by the fission yeast homologues of mammalian LSD1. PLoS ONE 2, e386 (2007).

32. Yamada, T., Fischle, W., Sugiyama, T., Allis, C. D. \& Grewal, S. I. The nucleation and maintenance of heterochromatin by a histone deacetylase in fission yeast. Mol. Cell 20, 173-185 (2005).

33. Noma, K., Allis, C. D. \& Grewal, S. I. Transitions in distinct histone H3 methylation patterns at the heterochromatin domain boundaries. Science 293, 1150-1155 (2001).

34. Thon, G., Bjerling, P., Bunner, C. M. \& Verhein-Hansen, J. Expression-state boundaries in the mating-type region of fission yeast. Genetics 161, 611-622 (2002).
35. Beach, D. H. Cell type switching by DNA transposition in fission yeast. Nature 305, 682-688 (1983).

36. Thon, G. \& Klar, A. J. The clr1 locus regulates the expression of the cryptic mating-type loci of fission yeast. Genetics 131, 287-296 (1992).

37. Thon, G., Cohen, A. \& Klar, A. J. Three additional linkage groups that repress transcription and meiotic recombination in the mating-type region of Schizosaccharomyces pombe. Genetics 138, 29-38 (1994).

38. Volpe, T. A. et al. Regulation of heterochromatic silencing and histone H3 lysine-9 methylation by RNAi. Science 297, 1833-1837 (2002).

39. Martienssen, R. \& Moazed, D. RNAi and Heterochromatin Assembly. Cold Spring Harb. Perspect. Biol. 7, a019323 (2015).

40. Grewal, S. I. \& Klar, A. J. A recombinationally repressed region between mat2 and mat3 loci shares homology to centromeric repeats and regulates directionality of mating-type switching in fission yeast. Genetics 146, 1221-1238 (1997).

41. Hansen, K. R., Ibarra, P. T. \& Thon, G. Evolutionary-conserved telomere-linked helicase genes of fission yeast are repressed by silencing factors, RNAi components and the telomere-binding protein Tazl. Nucleic Acids Res. 34, 78-88 (2006).

42. Buhler, M., Verdel, A. \& Moazed, D. Tethering RITS to a nascent transcript initiates RNAi- and heterochromatin-dependent gene silencing. Cell 125, 873-886 (2006).

43. Debeauchamp, J. L. et al. Chp1-Tas3 interaction is required to recruit RITS to fission yeast centromeres and for maintenance of centromeric heterochromatin. Mol. Cell. Biol. 28, 2154-2166 (2008).

44. Hall, I. M. et al. Establishment and maintenance of a heterochromatin domain. Science 297, 2232-2237 (2002).

45. Sadaie, M., Iida, T., Urano, T. \& Nakayama, J. A chromodomain protein, Chp1, is required for the establishment of heterochromatin in fission yeast. $E M B O \mathrm{~J}$. 23, 3825-3835 (2004).

46. Buscaino, A. et al. Raf1 Is a DCAF for the Rik1 DDB1-like protein and has separable roles in siRNA generation and chromatin modification. PLoS Genet. 8, e1002499 (2012).

47. Petrie, V. J., Wuitschick, J. D., Givens, C. D., Kosinski, A. M. \& Partridge, J. F. RNA interference (RNAi)-dependent and RNAi-independent association of the Chp1 chromodomain protein with distinct heterochromatic loci in fission yeast. Mol. Cell. Biol. 25, 2331-2346 (2005).

48. Ayoub, N., Goldshmidt, I., Lyakhovetsky, R. \& Cohen, A. A fission yeast repression element cooperates with centromere-like sequences and defines a mat silent domain boundary. Genetics 156, 983-994 (2000).

49. Jia, S., Noma, K. \& Grewal, S. I. RNAi-independent heterochromatin nucleation by the stress-activated ATF/CREB family proteins. Science 304, 1971-1976 (2004).

50. Grewal, S. I. \& Klar, A. J. Chromosomal inheritance of epigenetic states in fission yeast during mitosis and meiosis. Cell 86, 95-101 (1996).

51. Thon, G. \& Friis, T. Epigenetic inheritance of transcriptional silencing and switching competence in fission yeast. Genetics 145, 685-696 (1997).

52. Dodd, I. B., Micheelsen, M. A., Sneppen, K. \& Thon, G. Theoretical analysis of epigenetic cell memory by nucleosome modification. Cell 129, 813-822 (2007).

53. Dodd, I. B. \& Sneppen, K. Barriers and silencers: a theoretical toolkit for control and containment of nucleosome-based epigenetic states. J. Mol. Biol. 414, 624-637 (2011).

54. Micheelsen, M. A., Mitarai, N., Sneppen, K. \& Dodd, I. B. Theory for the stability and regulation of epigenetic landscapes. Phys. Biol. 7, 026010 (2010).

55. Sneppen, K. \& Dodd, I. B. A simple histone code opens many paths to epigenetics. PLoS Comput. Biol. 8, e1002643 (2012).

56. Xu, E. Y., Zawadzki, K. A. \& Broach, J. R. Single-cell observations reveal intermediate transcriptional silencing states. Mol. Cell 23, 219-229 (2006).

57. Canzio, D. et al. Chromodomain-mediated oligomerization of HP1 suggests a nucleosome-bridging mechanism for heterochromatin assembly. Mol. Cell 41, 67-81 (2011).

58. Schalch, T. et al. High-affinity binding of Chp1 chromodomain to K9 methylated histone $\mathrm{H} 3$ is required to establish centromeric heterochromatin. Mol. Cell 34, 36-46 (2009).

59. Tee, W. W. \& Reinberg, D. Chromatin features and the epigenetic regulation of pluripotency states in ESCs. Development 141, 2376-2390 (2014).

60. Morgan, H. D., Sutherland, H. G., Martin, D. I. \& Whitelaw, E. Epigenetic inheritance at the agouti locus in the mouse. Nat. Genet. 23, 314-318 (1999).

61. Angel, A., Song, J., Dean, C. \& Howard, M. A Polycomb-based switch underlying quantitative epigenetic memory. Nature 476, 105-108 (2011).

62. Angel, A. et al. Vernalizing cold is registered digitally at FLC. Proc. Natl Acad. Sci. USA 112, 4146-4151 (2015).

63. Berry, S., Hartley, M., Olsson, T. S., Dean, C. \& Howard, M. Local chromatin environment of a Polycomb target gene instructs its own epigenetic inheritance. eLife 4, e07205 (2015).

64. Hathaway, N. A. et al. Dynamics and memory of heterochromatin in living cells. Cell 149, 1447-1460 (2012). 
65. Al-Sady, B., Madhani, H. D. \& Narlikar, G. J. Division of labor between the chromodomains of HP1 and Suv39 methylase enables coordination of heterochromatin spread. Mol. Cell 51, 80-91 (2013).

66. Liu, N. et al. Recognition of $\mathrm{H} 3 \mathrm{~K} 9$ methylation by GLP is required for efficient establishment of $\mathrm{H} 3 \mathrm{~K} 9$ methylation, rapid target gene repression, and mouse viability. Genes Dev. 29, 379-393 (2015).

67. Motamedi, M. R. et al. Two RNAi complexes, RITS and RDRC, physically interact and localize to noncoding centromeric RNAs. Cell 119, 789-802 (2004).

68. Li, H. et al. An alpha motif at Tas3 C terminus mediates RITS cis spreading and promotes heterochromatic gene silencing. Mol. Cell 34, 155-167 (2009).

69. Li, F., Martienssen, R. \& Cande, W. Z. Coordination of DNA replication and histone modification by the Rik1-Dos2 complex. Nature 475, 244-248 (2011).

70. Alsing, A. K. \& Sneppen, K. Differentiation of developing olfactory neurons analysed in terms of coupled epigenetic landscapes. Nucleic Acids Res. 41, 4755-4764 (2013).

71. Petruk, S. et al. TrxG and PcG proteins but not methylated histones remain associated with DNA through replication. Cell 150, 922-933 (2012).

\section{Acknowledgements}

We thank Gilles Charvin for help with the microscopy at an early stage of the project, members of our groups for discussions and Janne Verhein-Hansen for technical assistance. We were funded by the Lundbeck Foundation, the Danish National Research Foundation (C-MOL Center), the Novo Nordisk Foundation, the Danish Council for Independent Research and the University of Copenhagen Center of Excellence MolPhysX.

\section{Author contributions}

M.J.O. and E.M.H.P. constructed strains, carried out the experiments and acquired images. K.S. performed the modelling. A.T. supervised the microscopy and performed the image analysis. G.T. designed the experiments and participated in strain constructions and microscopy. M.J.O., E.M.H.P. and A.T. participated in experimental design. K.S., A.T. and G.T. wrote the manuscript.

\section{Additional information}

Supplementary Information accompanies this paper at http://www.nature.com/ naturecommunications

Competing financial interests: The authors declare no competing financial interests.

Reprints and permission information is available online at http://npg.nature.com/ reprintsandpermissions/

How to cite this article: Obersriebnig, M. J. et al. Nucleation and spreading of a heterochromatic domain in fission yeast. Nat. Commun. 7:11518 doi: $10.1038 /$ ncomms11518 (2016).

(c) (i) This work is licensed under a Creative Commons Attribution 4.0 International License. The images or other third party material in this article are included in the article's Creative Commons license, unless indicated otherwise in the credit line; if the material is not included under the Creative Commons license, users will need to obtain permission from the license holder to reproduce the material. To view a copy of this license, visit http://creativecommons.org/licenses/by/4.0/ 